\title{
Progress in Developing a Low-Cost Large Deformable Mirror
}

\author{
Rikard Heimsten $^{a}$, Douglas G. MacMynowski ${ }^{b}$ and Torben Andersen ${ }^{a}$ \\ ${ }^{a}$ Lund Observatory, Lund University Box 43 SE-221 00 Lund, Sweden \\ ${ }^{b}$ Control and Dynamical Systems, California Institute of Technology 1200 E. California Blvd., \\ Pasadena, CA 91125
}

\begin{abstract}
Large $(>1 \mathrm{~m})$ deformable mirrors with hundreds or thousands of actuators are attractive for extremely large telescopes. Use of force actuators coupled to the mirror via suction cups, and electret microphones for position sensing, has the potential of substantially reducing costs. However, a mirror controlled with force actuators will have many structural resonances within the desired system bandwidth, shifting the emphasis somewhat of the control aspects. Local velocity and position loop for each actuator can add significant damping, but gives poor performance at high spatial frequencies. We therefore introduce a novel control strategy with many parallel "actuator families", each controlled by single-input-single-output controllers. This family approach provides performance close to that of global control, but without the accompanying robustness challenges. Using a complete simulation model of a representative large deformable mirror, we demonstrate feasibility of the approach.

This paper describes the challenges of non-ideal actuators and sensors. The results presented give an understanding of the required actuator bandwidth and the effects of the sensors dynamics. The conclusion is that the introduction of actuator and sensor dynamics does not limit the control system of the deformable mirror.
\end{abstract}

\section{INTRODUCTION}

Today's optical ground-based telescopes often have adaptive optics (AO) for compensation of atmospheric blurring and telescope aberrations. AO encompasses one or more deformable mirrors (DMs), controlled by numerous actuators, to compensate for the distortions in the wavefront of the light. Existing DMs typically have a diameter of tens of centimeters or less, but much larger DMs with sizes of 1-4 m are highly attractive for future Extremely Large Telescopes. Large DMs enable integration of the DMs into the optical telescope, avoiding use of post-focus relay optics.

Deformable mirrors up to about $0.9 \mathrm{~m}$ exist today. DMs based upon piezo-electric, electrostrictive or magnetostrictive actuators largely operate in a quasi-static mode. The DM is effectively constrained at each actuator location and the actuators are stiff "position actuators". In contrast, in a DM with soft force actuators the dynamics of the mirror structure enter directly into the control loops, thus making it more difficult to control. The reason is, the reaction mass is attached to the structure by a spring, thus the actuator has no authority over the structural modes.

The benefit of using soft actuators is that the mechanical tolerances can be more relaxed compared to the stiff actuators, potentially highly reducing the construction costs of large DMs. This paper advances the work presented in. ${ }^{1-3}$ In the latter, a control strategy was developed for perfect actuators and sensors. The effects of limited actuator and sensor bandwidth are presented in this paper.

The next section describes the control approach, including the rate and the position feedback loops, and the predicted performance is presented. The effects of limited actuator bandwidth are discussed in Section 3 and the effects of limited sensor bandwidth are discussed in Section 4.

Adaptive Optics Systems II, edited by Brent L. Ellerbroek, Michael Hart, Norbert Hubin, Peter L. Wizinowich, Proc. of SPIE Vol. $7736,77365 N$ - @ 2010 SPIE · CCC code: 0277-786X/10/\$18 - doi: 10.1117/12.855054 


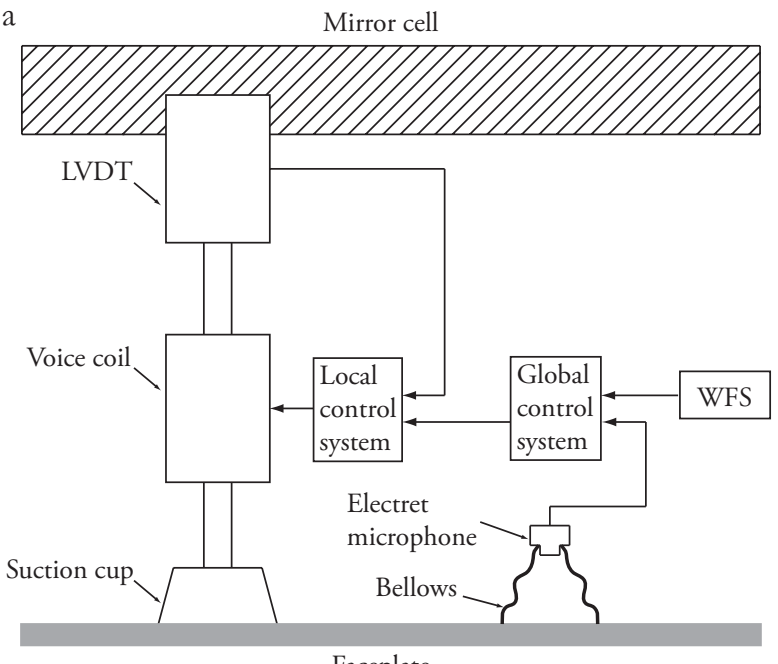

$\mathrm{b}$

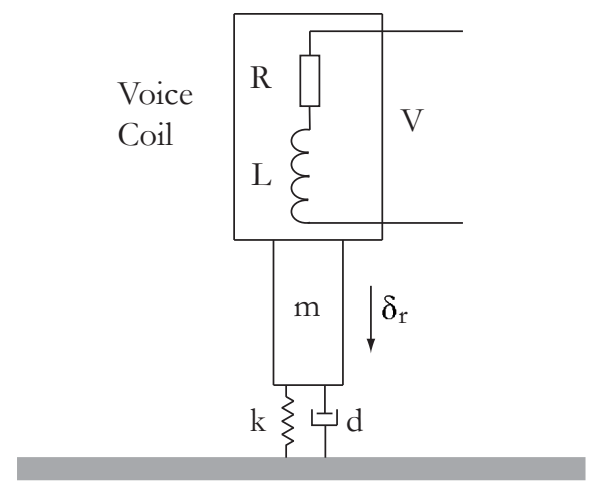

Figure 1. (a): Sensor scheme for the local and global controllers (b): Graphical illustration of a mathematical model of an actuator. Here $\delta_{r}$ is the displacement of the rod, $m$ the mass of the rod, $k$ the stiffness of the rod, $c$ the viscous damping coefficient of the rod, $L$ the inductance of the coil, $R$ the resistance in the coil and $V$ the voltage over the coil.

\section{BACKGROUND}

\subsection{Problem description}

The objective is to develop a low-cost concept for a large DM with force actuators attached to the back of the mirror through suction cups. Use of this actuator type in combination with a thin mirror leads to a poorly damped system with structural resonances within the desired control bandwidth. The wavefront sensor loop of the external adaptive optics system is insufficient to confront the problem with resonance frequencies. Thus, an additional feedback loop is constructed, using sensors attached to the back of the mirror, see Fig. 1a. The plan is to use electret microphones in bellows, non-collocated with the actuators, at the back of the mirror to sense mirror deflection.

\subsection{Mathematical model}

A mathematical model of the DM has been set up to develop and test a control strategy for the global control of the mirror. Hence, a finite element model of the mirror was established, using the software package Comsol Multiphysics, by approximating the mirror with a plane shell. The mesh includes about 5000 nodes, and each node has six degrees of freedom (DoF), i.e. translation along three mutually perpendicular axes and rotation around the same axes. The dynamic behavior of the structure is described by the differential equation

$$
\mathbf{M} \frac{\mathrm{d} \delta 2}{\mathrm{~d}^{2} \mathrm{t}}+\boldsymbol{\Lambda} \frac{\mathrm{d} \boldsymbol{\delta}}{\mathrm{dt}}+\mathbf{K} \boldsymbol{\delta}=\mathbf{f}
$$

where $\mathbf{M}, \boldsymbol{\Lambda}, \mathbf{K}$ are respectively the mass, damping and stiffness matrices, $\mathbf{f}$ is a force vector and $\boldsymbol{\delta}$ a vector holding angular and translation displacements. The example used throughout this study is a $2 \mathrm{~mm}$ thick, $1 \mathrm{~m}$ in diameter faceplate fixed in its center and made of the material Borosilicate. The actuators, in total 372, are placed in a square topology with an actuator pitch of $42 \mathrm{~mm}$. The sensors, in total 702, are each located between two adjacent actuators, i.e. in the general case each actuator has four neighbouring sensors. The use of a non-collocated sensor and actuator scheme gives rise to a phase lag, which is tolerable as long as the distance between the actuators and sensors is no more than $22 \mathrm{~mm} .{ }^{3}$ For actuator feedback loops, the average position signals from the four nearest sensors are used.

The full model of the DM is computationally impractical. Hence, model reduction has been performed as following: 
- Guyan reduction is used to reduce the number of DoFs to three for each node, retaining the out-of-plane translation and the two in-plane rotations.

- Modes with eigenfrequencies above $500 \mathrm{~Hz}$ are removed by modal truncation. Mode acceleration is applied to include the static contribution from the modes omitted by the truncation.

\subsection{Control system}

The frequency response from force to position for the poorly damped structure is shown in Fig. 2a. The poorly damped resonances are obstacles for designing the position loop directly, thus a control system which divides the feedback into rate and position loops has been designed as shown in Fig. $3 .^{3}$ The rate feedback servo is a proportional controller, $\mathbf{K}_{\mathbf{V}}$, and the position feedback servo is a integral controller, $\mathbf{K}_{\mathbf{I}}$, with a cross-over frequency of $250 \mathrm{~Hz}$.

The task of the rate feedback is to add damping to the faceplate, which is guaranteed if the matrix $\mathbf{K}_{\mathbf{V}}$ is positive definite. If the gain for the loop is tuned correctly, waves propagating away from an actuator can be dissipated before reaching the boundary and returning. The frequency response for the system with all rate loops closed is shown in Fig $2 \mathrm{~b}$. The matrix $\mathbf{K}_{\mathbf{V}}$ is diagonal which means that the controllers are local because every actuator interacts only with its nearest sensors using a single-input-single-output proportional controller. A diagonal matrix with only positive elements is positive definite, thus energy is dissipated at every actuator location.

The task of the position feedback is to provide a good command response for the outer wavefront sensor loop. This cannot be done satisfactorily in a single-input-single-output manner due to substantial coupling between adjacent actuators. A force from one actuator only will give tip/tilt to the whole mirror. This can be understood by studying the condition number of the static plant, $\mathbf{F}$, which is of the order of 105 , i.e. there is much higher loop gain for low spatial frequencies compared to the high spatial frequencies. To reduce the effect of crosstalk between the actuators we apply a local feedback scheme, using a set of actuator families centered around any give actuator. All actuators in a family, $\Omega$, are controlled simultaneously with forces that are proportional to a command signal, and the distribution of forces among actuators is selected to minimise the cost function

$$
J=\left\|\mathbf{K}^{-1} \mathbf{f}-\delta\right\|_{2}
$$

where $\mathbf{f}$ is the force vector and $\delta$ is the displacement vector. The elements of $\delta$ and $\mathbf{f}$, when the $j$-th actuator is controlled, are chosen accordingly:

$$
\begin{array}{lll}
\delta_{i}=0 & \text { for } & i \neq j \\
\delta_{i}=1 & \text { for } & i=j \\
f_{i}=0 & \text { for } & i \notin \Omega \\
f_{i} \in \mathbb{R} & \text { for } & i \in \Omega
\end{array}
$$

The force pattern for each family is stored as rows in the family matrix $\mathbf{Q}$. The performance prediction for the DM, when $\Omega$ includes 21 actuators, with all rate and position loops closed, is shown in Fig. 2c. The condition number for the family matrix multiplied by the static plant

$$
\mathbf{Q} \times \mathbf{F}
$$

is around 2, i.e. a remarkable improvement compared to the static plant alone.

\section{NON-IDEAL ACTUATORS}

Actuators limit performance in many active structures, thus it is important to study the effects of non-ideal actuators. The actuator type foreseen here is a steerable-mass actuator as shown in Fig. 1a, which encompasses a voice coil, a movable rod and a linear variable differential transformer (LVDT). The LVDT senses the position of the rod, thus indirectly the force exerted on the faceplate due to the presence of a flexible suction cup. The state-space model of the actuators is explained in the Section 3.1, and how actuators with a limited bandwidth influences system performance is analysed in Section 3.2. 


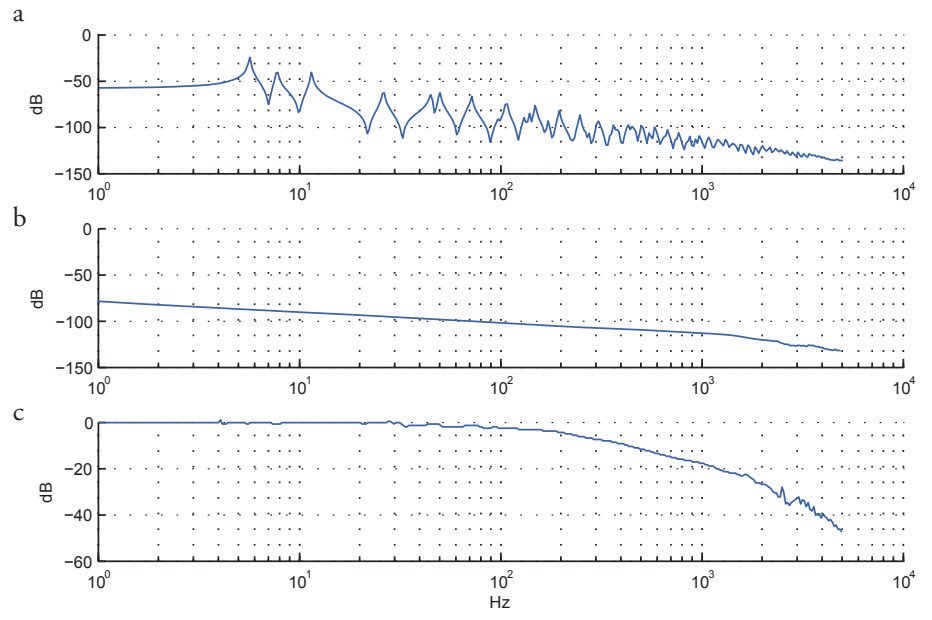

Figure 2. Frequency response for an actuator (a): open loop from force to position, (b): open loop from force to position with all rate feedback loops closed, (c): from command to position with all rate and position feedback loops closed and use of a family matrix controller.

\subsection{Mathematical model of the actuators}

The mathematical model of the actuator is illustrated in Fig. 1b, including symbol definitions. The spring connecting the actuator rod and the faceplate, $k$, represents the suction cup. The equation of motion, in the Laplace domain, is given by

$$
f=\left(m s^{2}+d s+k\right) \delta_{r}
$$

where the electromagnetic force developed by the voice coil is the product of the force constant and the current, $f=C i$. The current in the voice coil can be related to the voltage by

$$
\begin{array}{r}
c R i+L \frac{d i}{d t}=V-C_{e} \dot{\delta_{r}} \\
i=\frac{V-s C_{e} \delta_{r}}{R+s L}
\end{array}
$$

where $C_{e} \dot{\delta_{r}}$ is the back electromotive force due to the motion. ${ }^{4}$ The constant $C_{e}$ is the voice coil constant, describing how the current is related to the torque.

This is valid for a voltage input to the voice coil. If the inductance, $L$, of the voice coil winding is small, it can be neglected. If it is large enough to play a role, a local current loop can be added to suppress the influence of the inductance. Hence, for our simulations we can ignore the influence of the inductance. The previous expression then becomes

$$
i \approx \frac{V-s C_{e} \delta_{r}}{R}
$$

The transfer function from voltage to rod displacement, by inserting Eq.6 into Eq. 4, is given by:

$$
\frac{\delta_{r}}{V}=\frac{C}{R\left(m s^{2}+\left(\frac{C C_{e}}{G}+d\right) s+k\right)}
$$

The second order system in Eq. 7, indicates that the back electromotive force can be interpreted as additional damping. This can be guaranteed, up to a sufficiently high frequency, if a current controller is added to the amplifier. Further, Eq. 7 shows that the corner frequency of the actuator is set by the spring constant $k$ and the mass $m$ of the moving actuator rod, which is about a few Hertz. A local servo loop, with proportional gain $g$, 


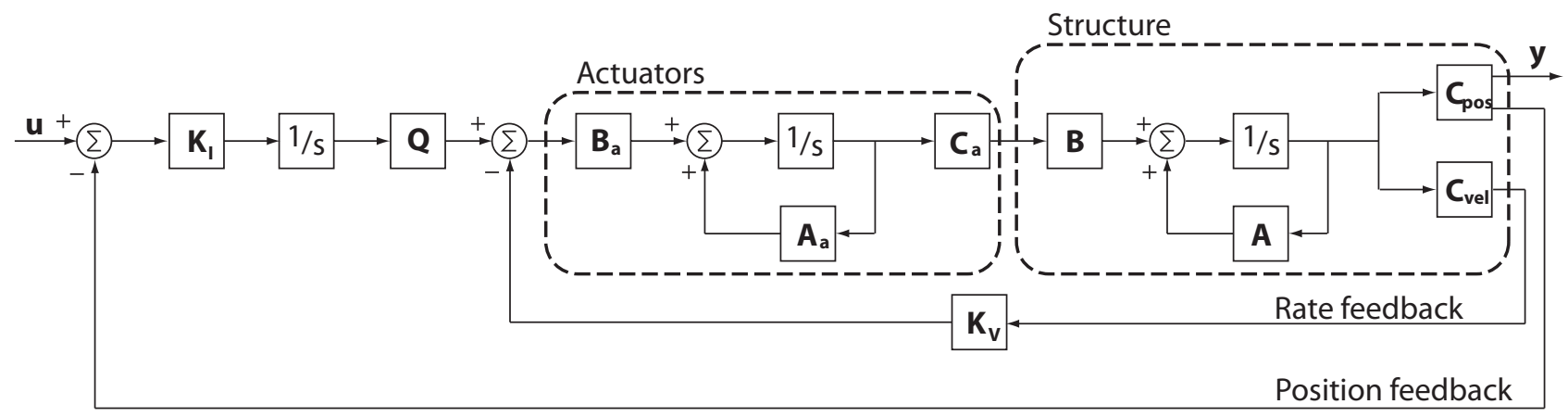

Figure 3. The block diagram includes the state-space model of the structure, the state-space model of the actuators and the rate and position feedback loops.

with feedback from the LVDT is added to increase the bandwidth of the actuator. The transfer function from voltage to rod displacement is changed according to

$$
\frac{\delta_{r}}{V}=\frac{C}{R\left(m s^{2}+\left(\frac{C C_{e}}{G}+d\right) s+g C+k\right)}
$$

i.e. additional stiffness is added. The natural frequency, $\omega_{a}$, and the damping, $\zeta_{a}$, of the second-order system in Eq. 8 is given by:

$$
\begin{gathered}
\omega_{a}=\sqrt{\frac{g C+k}{m}} \\
\zeta_{a}=\frac{C C_{e}+R d}{2 G \sqrt{m(g C+k)}}
\end{gathered}
$$

The dynamic features of the actuators described by Eq. 8 is integrated in the full mirror model through a state-space realisation, given by:

$$
\mathbf{A}_{\mathbf{a}}=\left[\begin{array}{cc}
-2 \zeta_{a} \omega_{a} & -\omega_{a}^{2} \\
1 & 0
\end{array}\right] \quad \mathbf{B}_{\mathbf{a}}=\left[\begin{array}{c}
\omega_{a}^{2} \\
0
\end{array}\right] \quad \mathbf{C}_{\mathbf{a}}=\left[\begin{array}{ll}
0 & 1
\end{array}\right] \quad \mathbf{D}_{\mathbf{a}}=0 \quad \mathbf{x}=\left[\begin{array}{c}
\dot{\delta} \\
\delta
\end{array}\right]
$$

The block diagram over the full system, including the state-space model for the actuators, is shown in Fig. 3.

\subsection{Influence of finite actuator bandwidth on system performance}

Use of an actuator with a limited bandwidth is more of a concern for the velocity loop than for the position loop. The velocity loop has a bandwidth at least equal to the lowest frequency at which the structural resonances flow together, which for a $1 \mathrm{~m}$ borosilicate mirror with a thickness of $2 \mathrm{~mm}$ is around $1 \mathrm{kHz}$ as can be seen from Fig. 2a. The position loop needs to have a bandwidth larger than $100 \mathrm{~Hz}$ as shown in Fig. 2c to satisfy a phase lag criterion for operation of adaptive optics.

The damping of the mirror structure has a major effect on the necessary bandwidth of the actuators. This can be understood by studying Fig. 2a. At a sufficiently high frequency, the response is no longer dominated by individual modes, instead there are enough modes participating at every frequency, to smooth out the peaks and anti-peaks in the frequency response. The starting point of this range of acoustic behavior can mathematically be explained for a plate, without boundary conditions, by the following steps. The modal density for a thin plate can be written $\mathrm{as}^{5}$

$$
N=\frac{\omega A}{2 \pi c_{g} c}
$$

where $\omega$ is the natural frequency, $A$ the plate area, $c_{g}$ the group velocity, and $c$ is the phase velocity. For bending vibrations in a thin plate, the phase velocity is ${ }^{5}$

$$
c=\sqrt{\omega}\left(\frac{D}{\rho h}\right)^{1 / 4}
$$


a
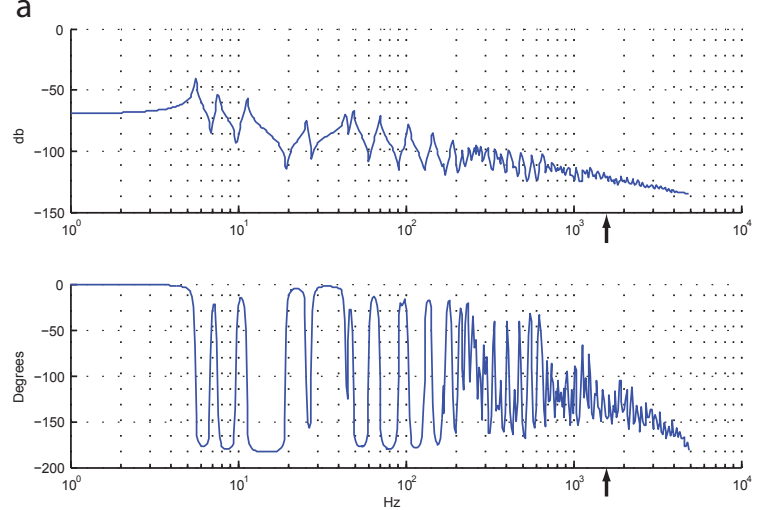

b
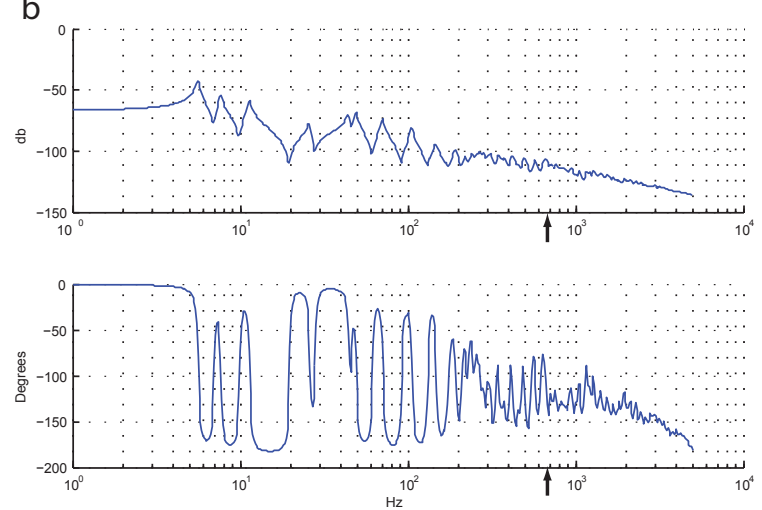

Figure 4. Frequency response from force to position for the structure. (a): with a modal damping of 0.01 , (b): with a modal damping of 0.03 . The arrows indicate approximately where the acoustic limit starts.

where $D$ is the flexural rigidity of the plate, $h$ is thickness and $\rho$ the density. The group velocity is twice the phase velocity, so $c_{g}=2 c$. Inserting Eq. 10 into Eq. 9 results in

$$
N=\frac{A}{4 \pi} \sqrt{\frac{\rho h}{D}}
$$

Further, the average modal spacing is described by $\Delta \omega=1 / N$, and the half-power bandwidth of each mode is $2 \zeta f_{a}$, where $f_{a}$ is the undamped frequency and $\zeta$ is the modal damping. ${ }^{6}$ The starting point of the acoustic behavior is then given by

$$
f_{a}=\frac{2 n_{f}}{A \zeta} \sqrt{\frac{D}{\rho h}}
$$

where $n_{f}$ is the factor by which the half-power bandwidth needs to exceed the average modal spacing. Eq. 12 shows that $f_{a}$ is inversely proportional to the damping of the structure. Conclusively, if the damping of the structure for all the modes is doubled, the requirement for the bandwidth of the actuators is halved. This is illustrated in Fig. 4, where the two frequency responses from force to position with two different modal damping coefficients are plotted. The arrows indicate approximately where the acoustic behavior starts. The gain, $\mathbf{K}_{\mathbf{V}}$, of the rate feedback loop is the second important factor, which affects the requirement of the bandwidth of the actuators. The frequency response when $\mathbf{K}_{\mathbf{V}}$ is tuned to its maximum value is shown in Fig. $2 \mathrm{~b}$. However, the Bode plot does not necessarily need to look like that of Fig. $2 \mathrm{~b}$ as long as a position loop controller can be. Thus, the optimum $\mathbf{K}_{\mathbf{V}}$ is actually found for the minimum velocity loop gain at which a useful position loop can be designed.. The open loop system, which the position loops needs to control, is given by

$$
\mathbf{Q} \times \mathbf{F}_{\mathbf{V}}(\mathbf{s})
$$

where $\mathbf{F}_{\mathbf{V}}(\mathbf{s})$ is the transfer function matrix of the plant with the Laplace operator $s$, including the closed rate feedback loop and $\mathbf{Q}$ is the family matrix found by applying Eq. 2. The frequency response of $\mathbf{Q} \times \mathbf{F}_{\mathbf{V}}(\mathbf{s})$ for two different values of $\mathbf{K}_{\mathbf{V}}$ is shown in Fig. 5. As illustrated, the gain of the rate feedback loop can be decreased with a factor of $1 / 5$, from its maximum value, without any significant effect below $250 \mathrm{~Hz}$ (the cross-over frequency of the integral controller in the position feedback servo).

Conclusively, when the system shown in Fig. 3 is evaluated with a structural damping of 0.01 for each eigenmode, then $\mathbf{K}_{\mathbf{V}}$ can be set to $1 / 5$ of its maximum value with an actuator damping of $\zeta_{a}=0.5$. The the actuator bandwidth should be chosen such that the stability of the feedback loops is not effected. This is achieved when the actuator bandwidth exceeds $2150 \mathrm{~Hz}$.

\section{NON-IDEAL SENSORS}

The non-collocated sensors placed on the back of the mirror, give feedback signal to the rate and the position loops. The electret microphones are placed within bellows, providing a closed environment and increased sensi- 
a
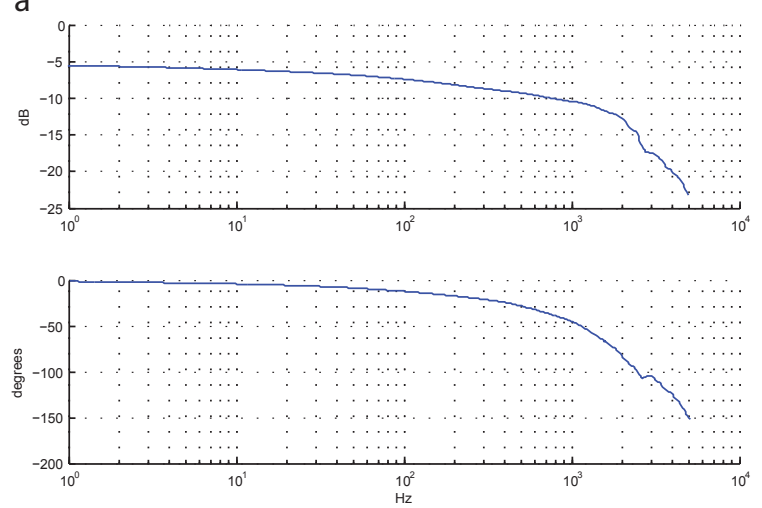

b
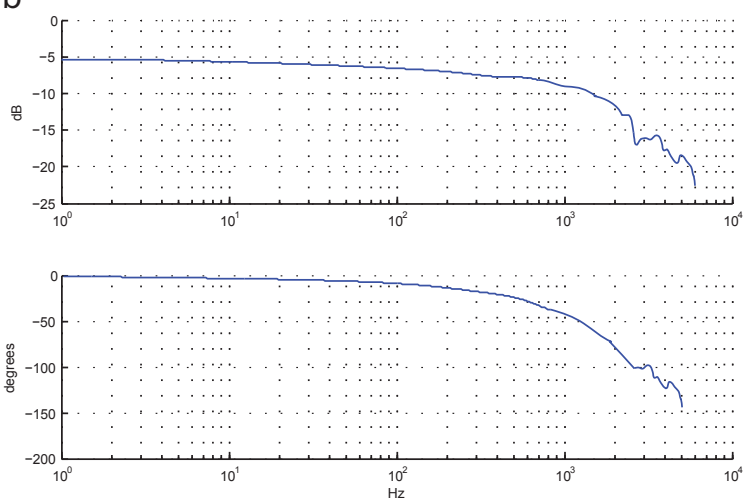

Figure 5. Frequency response from family force command to position without the position loop closed. (a): with the maximised $\mathbf{K}_{\mathbf{V}}$, (b): with a $\mathbf{K}_{\mathbf{V}}$ decreased by $80 \%$ of the maximum value.

tivity as shown in Fig. 1a. A slight preload is used to ensure contact between the bellows and the back of the mirror. A state-space model of the sensors is introduced in the Section 4.1 and the impact of the dynamics of the on the control system is presented in Section 4.2.

\subsection{Mathematical model of the sensors}

The electret microphones encompass two capacitors: a foil of a dielectric material and a metallic backplate. A cavity between the two capacitors forms a thin air gap. As the pressure over the dielectric material fluctuates, the air gap distance, $d$, varies because the foil has only little stiffness. Thus, the voltage, $V_{s}$ developed between the capacitors is described by

$$
V_{s}=\frac{Q d}{A_{m i c} e_{o}}
$$

where $e_{0}$ is the electric permittivity of free space, $A_{m i c}$ is the surface area and $Q$ is the charge around in dielectric material.

The pressure changes within the bellows are believed to be adiabatic, i.e. there is no heat transfer between the air and the bellows, and the ratio of the specific heat capacities is $\gamma=1.4$. The following equation is valid for an adiabatic gas

$$
p v^{\gamma}=\text { constant }
$$

where $p$ is the pressure and $v$ is the volume. The differential change of pressure due to differential volumetric change of the volume inside the bellows is

$$
\Delta \rho=\frac{\rho^{\prime} \gamma}{v^{\prime}} \Delta v
$$

using a linearisation of the variables around the equilibrium values $p^{\prime}$ and $v^{\prime}$. Eq. 14 is also valid for the pressure changes in the cavity between the electret foil and the backplate. Thus, a voltage change is proportional to the distance between the two capacitors, which is proportional to the pressure change at the electret foil, which is in turn proportional to the displacement of the mirror. This results in a static relationship between mirror displacement, $\delta_{m}$ and voltage change:

$$
\frac{V_{s}}{\delta_{m}}=k
$$

Eq. 15 is only valid for a limited frequency range. The electret microphones have zero response at DC, first because that is not required for use as microphones, and secondly because such a system is difficult to implement (it would effectively be a pressure transducer). There is also a roll off at high frequencies, normally around a few tens of $\mathrm{kHz}$. Conclusively, the electret microphone can be modeled as a combined high-pass filter with a corner 


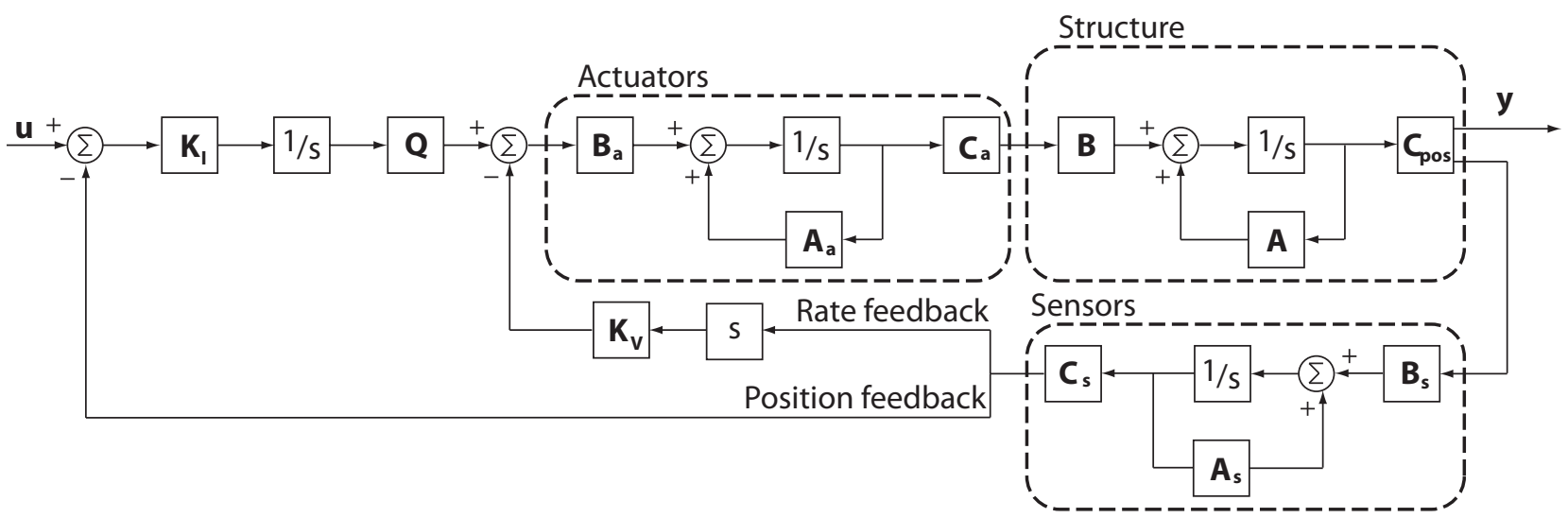

Figure 6. The block diagram includes the state-space model of the structure, the state-space model of the actuators, the state-space model of the electret microphones, and the rate and position feedback loops.

frequency, $\omega_{h p}$, at $20 \mathrm{~Hz}$ and a low-pass filter with a corner frequency, $\omega_{l p}$, at $20 \mathrm{kHz}$., and with a flat response between the cut-off frequencies. The state-space model is given by:

$$
\mathbf{A}_{\mathbf{s}}=\left[\begin{array}{cc}
0 & -\omega_{h p} \omega_{l p} \\
1 & -\omega_{h p}-\omega_{l p}
\end{array}\right] \quad \mathbf{B}_{\mathbf{s}}=\left[\begin{array}{c}
0 \\
\omega_{l p}
\end{array}\right] \quad \mathbf{C}_{\mathbf{s}}=\left[\begin{array}{ll}
0 & 1
\end{array}\right] \quad \mathbf{D}_{\mathbf{s}}=0 \quad \mathbf{x}=\left[\begin{array}{c}
\dot{\delta} \\
\delta
\end{array}\right]
$$

The block diagram of the system, including the dynamics of the electret microphones, is shown in Fig. 6 .

\subsection{Influence of limited sensor bandwidth on system performance}

The effects of using the non-ideal sensors relate to the low-pass and the the high-pass filter parts. The corner frequency of the former must match the required bandwidth of the actuator, thus a flat frequency response up to about $2500-3000 \mathrm{~Hz}$ is needed. The limit is not set by the electret microphones themselves, but by the dynamics in the rubber bellows enclosing the electret microphones. This needs to be investigated further.

The low sensor response below $20 \mathrm{~Hz}$ is a problem, foremost in the rate feedback loop because there are structural resonance and anti-resonance peaks below $20 \mathrm{~Hz}$, as shown in Fig. 2a. No electronical damping will be added to these. However, it turns out that use of the local family approach already introduced is beneficial in this respect because structural resonances are not excited by the control system, so that stability issues do not become a concern. The frequency response from force to position in Fig. 7, is the open-loop system for the state-space model of the structure multiplied with the family matrix. The large effect of the family matrix at low frequencies is illustrated when comparing Fig. 7 and Fig. 2a. Besides decoupling adjacent actuators from each other, the family matrix also ensures that the resonance and anti-resonance peaks below $30 \mathrm{~Hz}$ are not excited by actuator commands.

The frequency response from force to true position of the system in Fig. 6 is shown in Fig. 7. The output of the system differs significantly from that shown in Fig. 2c below $20 \mathrm{~Hz}$, because of the decreasing response of the electret microphones. Use of an integral controller of the position loop affects the system performance at low frequencies. This is not a problem in a complete AO-system, since the feedback signal from the wavefront sensor covers this frequency region.

\section{CONCLUSIONS}

We have evaluated the bandwidth requirements for non-ideal actuators and sensors based upon stability considerations. The control system has position and rate feedback, and it is the rate feedback which determines the requirements for the actuator and sensor dynamics.

The actuator bandwidth needs to exceed the lower limit frequency for acoustic behavior, described by Eq. 12 for an unconstrained plate. The lower limit frequency for acoustic behavior is inversely proportional to structural damping, which makes it difficult to accurately predict the actuator bandwidth required. As can be seen from 

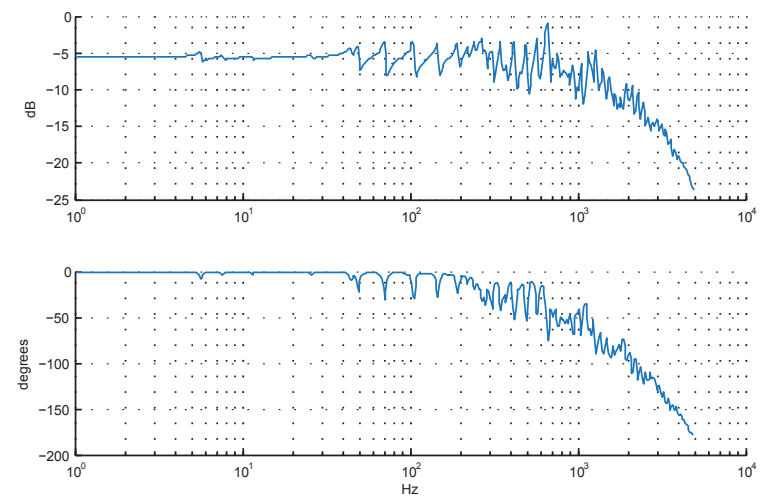

Figure 7. Open-loop frequency response from force to position for the family matrix multiplied with the plant.
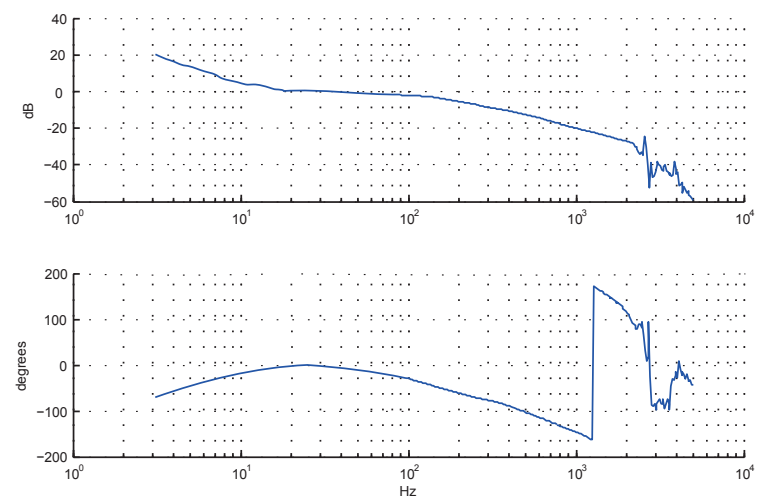

Figure 8. Frequency response from force to position for the full system, including actuator and sensor dynamics. The region bellow $20 \mathrm{~Hz}$ is where the electret microphones have started to roll off.

Eq. 12, there is an inverse relationship between the starting point of the acoustic behavior and the area of the plate, i.e. the requirements to the actuator bandwidth are less severe for a large DM.

The response of the electret microphones drops off at low and high frequencies, modeled as first-order highpass and low-pass filters. The corner frequency of the low-pass filter must exceed the actuator bandwidth to ensure stability. Most electret microphones have a flat response up to $10-20 \mathrm{kHz}$ but it should be ensured that structural effects in the bellows do not limit the bandwidth excessively. The influence of mirror eigenmodes below $20 \mathrm{~Hz}$, where the response of the electret microphones drops off, is efficiently suppressed by use of the family matrix in the position loop. Then, the feedback signal from the wavefront sensor is sufficient to close to position loop.

\section{REFERENCES}

[1] T. Andersen, O. Garpinger, M. Owner-Petersen, F. Bjoorn, R. Svahn and A. Ardeberg Noval concept for large deformable mirrors Optical Engineering Vol. 452006

[2] R. Heimsten, T. Andersen, M. Owner-Petersen Modeling Large Deformable Mirrors J. Proceedings of the SPIE Vol. 70172008

[3] D. MacMynowski, R. Heimsten and T. Andersen Distributed Force Control of Deformable Mirrors European Journal of Control Submitted

[4] A. Preumont Vibration Control of Active Structures ISBN1-4020-0496-6 2002

[5] R.H. Lyon and R.G. DeJong Theory and application of statistical energy analysis ISBN 07506911151995

[6] R.H. Lyon Machinery noise and diagnostics ISBN 04099010161986 